\title{
Hypercalcemia of malignancy treated with cinacalcet
}

\author{
Nikolaos Asonitis,1,2*, Eva Kassi,1,2*, Michalis Kokkinos, Ilias Giovanopoulos', Foteini Petychaki \\ and Helen Gogas ${ }^{1}$ \\ ${ }^{1}$ National and Kapodistrian University of Athens, First Department of Internal Medicine, Laikon Hospital, School of \\ Medicine, Athens, Greece and 2Msc Metabolic Bone Diseases, National and Kapodistrian University of Athens, School \\ of Medicine, Athens, Greece \\ *(N Asonitis and $\mathrm{E}$ Kassi contributed equally to this work)
}

\author{
Correspondence \\ should be addressed \\ to E Kassi \\ Email \\ ekassi@med.uoa.gr
}

\section{Summary}

Hypercalcemia of malignancy is the most common cause of hypercalcemia in hospitalized patients. It is associated with a poor prognosis, since it reflects an advanced cancer stage. Among all cancer in females, breast cancer is the most common malignancy, and it has the highest prevalence of hypercalcemia. Approximately $70 \%$ of patients with breast cancer have bone metastases and $10 \%$ of them will have hypercalcemia as a complication at some point in the disease. Herein, we report a 69-year-old female patient with metastatic breast cancer, who developed severe hypercalcemia in the course of her disease and was diagnosed with humoral hypercalcemia of malignancy (HHM). Intense hydration along with corticoisteroids and antiresorptive medication (calcitonin, bisphosphonates and denosumab) were administered to the patient. Despite the above treatment, serum calcium levels remain elevated and calcimimetic cinacalcet was added. Upon discontinuation of cinacalcet, calcium levels were raised and returned back to the normal levels following re-initiation of the calcimimetic. Her calcium level restored to normal, and she was discharged with the following medical treatment: denosumab monthly, and cinacalcet at a titrated dose of $90 \mathrm{mg}$ per day. The patient is followed as an outpatient and 11 months later, her calcium level remained within the normal range.

\section{Learning points:}

- Hypercalcemia of malignancy is the most common cause of hypercalcemia in hospitalized patients.

- Breast cancer has the highest prevalence of hypercalcemia.

- The cornerstone of therapy remains the intense hydration and intravenous bisphosphonates (preferably zoledronic acid).

- In case of persistent hypercalcemia of malignancy, the administration of calcimimetic cinacalcet could be an additional effective therapeutic option.

\section{Background}

Hypercalcemia of malignancy affects up to $20 \%$ of cancer patients during the course of their disease (1). The majority of cases are humoral in etiology and related to parathyroid hormone-related protein (PTHrP) (2).The treatment of HHM remains a challenge. There are various agents such as calcitonin, bisphosphonates (BPs) and denosumab, which can be administered in addition to the intense hydration; however, there are cases that these agents can be ineffective.

In such cases of persistent HHM, the administration of calcimimetic cinacalcet could be an additional effective therapeutic option, although the exact mechanism of this action remains to be elucidated. 


\section{Case presentation}

A 69-year-old female was admitted to the hospital with severe hypercalcemia (serum calcium level: $15.2 \mathrm{mg} / \mathrm{dL}$ ). Her symptoms were muscle weakness, nausea, vomiting, constipation, polyuria and malaise. Upon physical examination, she was found to have a dry oral mucosa with loss of skin turgor; she was afebrile, with normal blood pressure and tachycardia (114 bpm). Electrocardiography showed shortening of the QT interval. Results from the initial laboratory investigation on admission are shown on Table 1.

According to her medical history, she was treated for hypothyroidism receiving levothyroxine $100 \mu \mathrm{g} /$ day, and 6 years ago, she was diagnosed with breast cancer of the left mammary gland. She was submitted to total left mastectomy. A biopsy specimen revealed an infiltrating ductal and lobular mammary carcinoma with diameter $4.7 \mathrm{~cm}$, grade II, ER (+), PR (-), cerb-2 (-) and Ki67 30\%. Following mastectomy, the patient was submitted to chemotherapy, radiotherapy and hormonal therapy with fulvestrant. Three years later, she was diagnosed with bone metastases of the thoracic and lumbar spine and pelvis and zolendronic acid i.v monthly for a year and radioactive samarium (153Sm-EDTMP), were administered. Zolendronic acid was last administered two years before her admission. The biochemical profiles of the patient one year before admission as well as on admission are shown on Table 1.

\section{Investigation}

Although, in our case, there was an established malignancy with a history of bone metastases, intact PTH was checked to assess a possible coexistent of primary hyperparathyroidism (PHPT) or the rare possibility of ectopic PTH secretion, and it was found suppressed.

Taking into account the low levels of PTH, and based on the rest biochemical and hormonal profile (Fig. 1) characterized by a $1,25(\mathrm{OH})_{2} \mathrm{D}$ concentration within the normal range, and low serum phosphorus level, the most likely diagnosis was pure HHM caused by increased PTHrP production. In our patient, measurement of PTHrP was not feasible but in patients with the above findings, the diagnosis of HHM would be made even in the absence of the PTHrP measurement (3).

It should be mentioned that, although $1,25(\mathrm{OH})_{2} \mathrm{D}$ may be elevated in patients with PHPT, this is not the case in patients with humoral hypercalcemia.

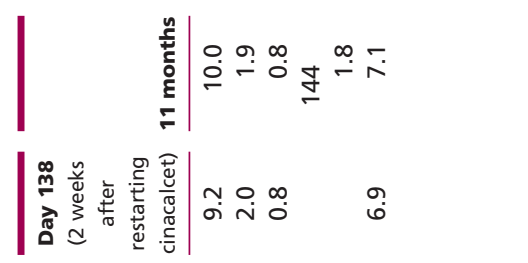

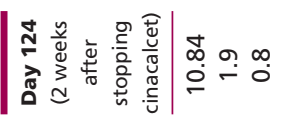

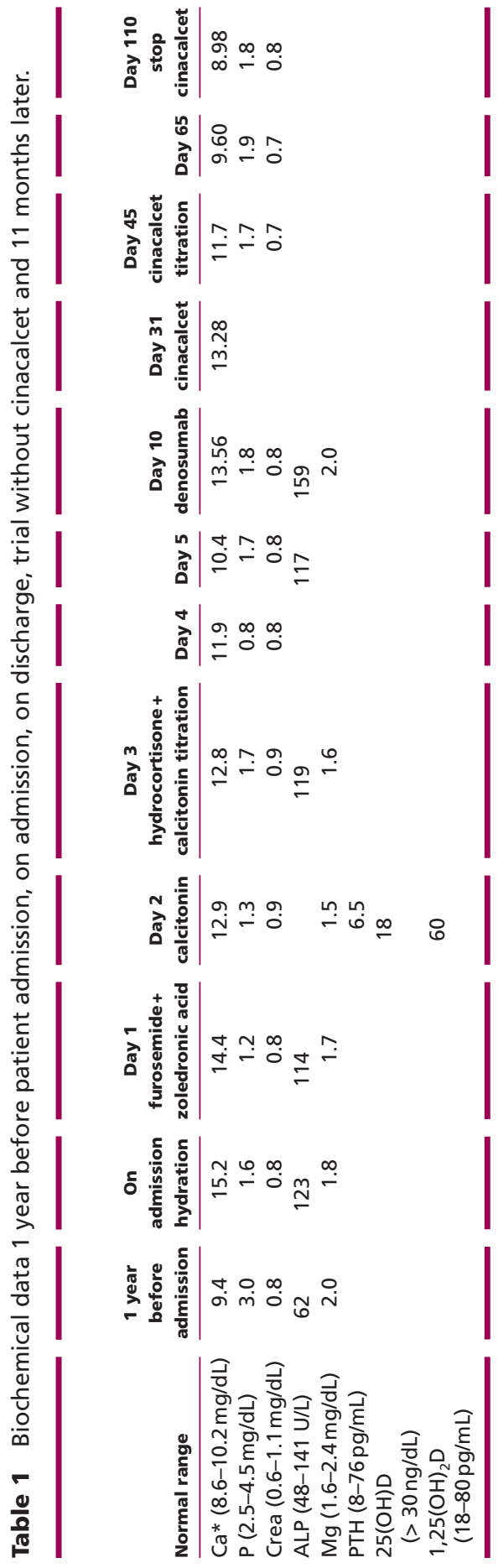

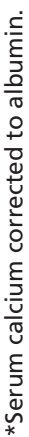




\begin{tabular}{|c|c|c|c|}
\hline & $\begin{array}{l}\text { Humoral } \\
\text { Hypercalcemia }\end{array}$ & $\begin{array}{l}\text { Osteolytic } \\
\text { hypercalcemia }\end{array}$ & $\begin{array}{l}\text { 1,25(OH)D - } \\
\text { mediated } \\
\text { hypercalcemia }\end{array}$ \\
\hline Calcium & 1 & 1 & 1 \\
\hline Phosphorus & $\sqrt{2}$ & 个 & 1 \\
\hline PTH & 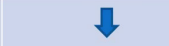 & $\checkmark$ & 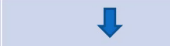 \\
\hline${ }_{25}(\mathrm{OH}) \mathrm{D}$ & $\Leftrightarrow$ & $\Leftrightarrow$ & $\Leftrightarrow$ \\
\hline $1.25(\mathrm{OH})_{2} \mathrm{D}$ & $\Leftrightarrow$ & $\Leftrightarrow$ & 个 \\
\hline PTHrP & 1 & 8 & 8 \\
\hline
\end{tabular}

\section{Figure 1}

Differential diagnosis of hypercalcemia in our patient with malignancy.

\section{Treatment}

Treatment was initiated upon admission aiming at the decreasing serum calcium levels. 1.5 L IV of normal saline bolus was administered followed by maintenance of fluids at $100-150 \mathrm{~mL} / \mathrm{h}$ titrated to a urine output of $100 \mathrm{~mL} / \mathrm{h}$. A total of $4 \mathrm{~L}$ of normal saline over the first $24 \mathrm{~h}$ was administered and continued with maintenance of fluids until she was euvolemic. The loop diuretic furosemide $40 \mathrm{mg}$ every $12 \mathrm{~h}$ was also administered IV to increase renal calcium excretion and zoledronic acid $4 \mathrm{mg}$ was administered IV on the first day. On the second day, serum calcium levels decreased to $12.9 \mathrm{mg} / \mathrm{dL}$. Thereafter, calcitonin 4 units/kg every $12 \mathrm{~h}$ subcutaneously (S.C.) was administered with dose titration to 8 units/kg along with hydrocortisone $50 \mathrm{mg}$ every $6 \mathrm{~h}$ IV. On the fifth day, calcium level was $10.4 \mathrm{mg} / \mathrm{dL}$ and the patient was discharged with following instructions: methylprednisolone $16 \mathrm{mg}$ per os, furosemide $20 \mathrm{mg}$ every twelve hours per os, while she was instructed to reduce calcium intake and to increase intake of water (at least $3 \mathrm{~L}$ water per day). Five days later, serum calcium levels increased to $13.56 \mathrm{mg} / \mathrm{dL}$. Denosumab $120 \mathrm{mg}$ S.C. weekly was added in the treatment of patient. Three weeks later, calcium levels were still elevated (calcium $=13.28 \mathrm{mg} / \mathrm{dL}$ ) and cinacalcet $60 \mathrm{mg} /$ day was added to her medication. After two weeks, calcium levels were decreased to $11.7 \mathrm{mg} / \mathrm{dL}$ and cinacalcet was titrated up to $90 \mathrm{mg}$ per day, while denosumab was administrated monthly. Three weeks later calcium was normalized $(9.6 \mathrm{mg} / \mathrm{dL})$.

\section{Outcome and follow-up}

Calcium levels maintained at normal range during the next 1.5 months (Table 1). To confirm its efficacy regarding the hypocalcemic effect on the hypercalcemia of malignancy treatment with cinacalcet was stopped, and 14 days later, serum calcium level increased by $1.7 \mathrm{mg} / \mathrm{dL}$. The patient 11 months after her admission is well and her calcium levels remain stable.

\section{Discussion}

Hypercalcemia of malignancy is the main cause of hypercalcemia in cancer patients and occurs in almost $20 \%$ of them during the course of their disease $(1,2)$. It can be attributed to (1) direct osteolytic bone metastasis, (2) humoral mechanisms mediated by parathyroid hormonerelated peptide (PTHrP), (3) increased extrarenal synthesis of activate $1,25[0 \mathrm{H}]_{2} \mathrm{D}$ or (4) rarely ectopic production and secretion of PTH (4). Humoral hypercalcemia is most commonly presented in renal cell, squamous cell and ovarian cancers while breast cancer is usually characterized by hypercalcemia due to osteolytic bone lesion (1).

PTHrP is responsible for the majority of case of HHM and occurs in $33-84 \%$ of patients with breast cancer (5). PTHrP, a hormone produced and secreted by cancer cells, can bind the common PTH/PTHrP receptor mimicking the effects of PTH and activating thus the osteoclastic bone resorption. However, PTHrP unlike PTH does not increase 1a hydroxylase activity so that increased circulating $1,25(\mathrm{OH})_{2} \mathrm{D}$ is not seen in patients with PTHrP-dependent HHM.

The treatment comprises the aggressive IV hydration, while BPs administration are the mainstay of treatment (6). In our case, despite the intense hydration and the addition of furosemide following volume expansion, calcium levels remained elevated. Actually, volume expansion can lead to a decrease in serum calcium of approximately $2 \mathrm{mg} / \mathrm{dL}$ (6).

Calcitonin is a weak agent with a rapid onset of action which interferes with osteoclast maturation and promotes calciuresis. Hence, we added it until the effects of BPs become apparent (7). Since the combination therapy with calcitonin and corticosteroids has been shown to prevent calcitonin tachyphylaxis, corticosteroids were added to our patient's treatment, although she was not suffered from a steroid-sensitive tumor such as multiple myeloma, leukemia or lymphoma where corticoisterois are the therapy of choice.

Zoledronic acid, which is the preferred BPs in the hypercalcemia of malignancy, was given on the first day of her admission to the hospital, as a first-line therapy (6). Four to ten days later - when BPs usually exert their maximum effects, serum calcium was still elevated. It should be noted that resistance to BPs may occur in 
humoral HCM since bisphosphonates are unable to inhibit PTHrP-induced calcium reabsorption in the kidneys.

Denosumab is a human monoclonal antibody to RANKL that inhibits osteoclasts activity and bone resorption. In 2014, it was approved for hypercalcemia refractory to bisphosphonates therapy while studies have shown that denosumab was more efficacious than zoledronic acid in delaying or preventing hypercalcemia of malignancy in patients with advanced cancer including breast cancer (8). Denosumab dosed as $120 \mathrm{mg}$ subcutaneously weekly for the first month and monthly thereafter, it lowered calcium level in $60 \%$ of patients within 10 days, and it had a median duration of 104 days.

In our patient, three weeks later, calcium levels were still elevated; thus, the addition of cinacalcet was decided. Cinacalcet is a calcimimetic agent that activates the calcium-sensing receptor (CaSR). Cinacalcet has recently been approved for the reduction of hypercalcemia in patients with parathyroid carcinoma, those with severe primary hyperparathyroidism (PHPT) who are unable to undergo parathyroidectomy and finally for the treatment of secondary hyperparathyroidism in patients with endstage renal disease on maintenance dialysis therapy (9). To date, one case has been reported regarding hypercalcemia of malignancy treated with cinacalcet, by Sternlicht and Glezerman (6). In this case, a patient with renal cell carcinoma and HHM was treated with BPs and denosumab with poor results and cinacalcet $60 \mathrm{mg}$ was added leading to normalization of serum calcium at 10 weeks. To our knowledge, this is the second case of HHM treated effectively with cinacalcet. This hypocalcemic effect could be mediated through CaSR. Of note, CaSR is expressed not only in the parathyroid glands and kidneys, but also in bone cells regulating bone homeostasis. In young animals, activation of CaSR in osteoclasts has been shown to inhibit bone resorption, resulting in increased bone anabolism (10). However, it should be noted that even in the absence of CaSR, cinacalcet found to attenuate PTHrP-mediated increase in serum calcium, in mice bearing Rice H-500 Leydig cell or C26-DCT colon tumors; interestingly, this effect was accompanied by increased plasma calcitonin (11).

The administration of PTHrP-related antibodies has been recently aroused as a promising therapy for the treatment of HHM. Until then, the administration of calcimimetic cinacalcet could be an additional effective therapeutic option.

\section{Declaration of interest}

The authors declare that there is no conflict of interest that could be perceived as prejudicing the impartiality of the research reported.

\section{Funding}

This research did not receive any specific grant from any funding agency in the public, commercial or not-for-profit sector.

\section{Author contribution statement}

H G was the physician of the patient. N A, E K, M K, I G and F P were taking care of the patient during the hospitalization. $\mathrm{N} \mathrm{A}$ and $\mathrm{E} K$ wrote the manuscript. All authors read, edited and approved the case report.

\section{References}

1 Stewart AF 2005 Clinical practice. Hypercalcemia associated with cancer. New England Journal of Medicine 352 373-379. (https://doi. org/10.1056/NEJMcp042806)

2 Jung K \& Lein M 2014 Bone turnover markers in serum and urine as diagnostic, prognostic and monitoring biomarkers of bone metastasis. BBA 184 425-438. (https://doi.org/10.1016/j. bbcan.2014.09.001)

3 Lumachi F, Brunello A, Roma A \& Basso U 2009 Cancer-induced hypercalcemia. Anticancer Research 29 1551-1556.

4 Clines GA \& Guise TA 2008 Molecular mechanisms and treatment of bone metastasis. Expert Reviews in Molecular Medicine 10 e7. (https://doi.org/10.1017/S1462399408000616)

5 Grill V, Ho P, Body JJ, Johanson N, Lee SC, Kukreja SC, Moseley JM \& Martin TJ 1991 Parathyroid hormone-related protein: elevated levels in both humoral hypercalcemia of malignancy and hypercalcemia complicating metastatic breast cancer. Journal of Clinical Endocrinology and Metabolism 73 1309-1315. (https://doi.org/10.1210/jcem-73-6-1309)

6 Sternlicht H \& Glezerman IG 2015 Hypercalcemia of malignancy and new treatment options. Therapeutics and Clinical Risk Management 11 1779-1788. (https://doi.org/10.2147/TCRM.S83681)

7 Fatemi S, Singer FR \& Rude RK 1992 Effect of salmon calcitonin and etidronate on hypercalcemia of malignancy. Calcified Tissue International 50 107-109. (https://doi.org/10.1007/BF00298784)

8 Diel IJ, Body JJ, Stopeck AT, Vadhan-Raj S, Spencer A, Steger G, von Moos R, Goldwasser F, Feng A \& Braun A 2015 The role of denosumab in the prevention of hypercalcaemia of malignancy in cancer patients with metastatic bone disease. European Journal of Cancer 51 1467-1475, (https://doi.org/10.1016/j.ejca.2015.04.017)

9 Silvrberg SJ, Rubin MR, Faiman C, Peacock M, Shoback DM, Smallridge RC, Schwanauer LE, Olson KA, Klassen P \& Bilezikian JP 2007 Cinacalcet hydrochloride reduces the serum calcium concentration in inoperable parathyroid carcinoma. Journal of Clinical Endocrinology and Metabolism 92 3803-3808. (https://doi.org/10.1210/ jc.2007-0585)

10 Goltzman D \& Hendy GN 2015 The calcium-sensing receptor in bone-mechanistic and therapeutic insights. Nature Reviews Endocrinology 11 298-307. (https://doi.org/10.1038/nrendo.2015.30)

11 Colloton M, Shatzen E, Wiemann B, Starnes C, Scully S, Henley C \& Martin D 2013 Cinacalcet attenuates hypercalcemia observed in mice bearing either Rice H-500 Leydig cell or C26-DCT colon tumors. European Journal of Pharmacology 712 8-15. (https://doi. org/10.1016/j.ejphar.2013.04.013) 